\title{
Providing 24-hour child and adolescent mental health services: demand and outcomes
}

\author{
Patricia Byrne, ${ }^{1}$ Lorna Power, ${ }^{2}$ Carole Boylan, ${ }^{1}$ Mohammed lqbal, ${ }^{3}$ \\ Margo Anglim, ${ }^{1}$ Carol Fitzpatrick ${ }^{1,2}$
}

The Psychiatrist (2011), 35, 374-379, doi: 10.1192/pb.bp.110.033316

${ }^{1}$ Children's University Hospital, Dublin

${ }^{2}$ University College Dublin;

${ }^{3}$ Mater CAMHS, Dublin, Ireland

Correspondence to Patricia Byrne (patricia.byrne4@hse.ie)

First received 1 Nov 2010, final revision

13 Mar 2011, accepted 31 May 201
Aims and method The provision of 24-hour specialist child and adolescent mental health services (CAMHS) is a key target for service commissioners. However, a lack of data exist on models of service delivery or levels of need for out-of-hours specialist CAMHS to guide service development. We aim to describe a model of 24-hour service provision and provide information on the demand for and outcome of assessments of a service in Dublin, Ireland, using a 6-year retrospective case study design.

Results A total of 468 emergency presentations occurred during the study period; $80 \%$ presented with self-harm or suicidal ideation. Two-thirds presented outside of working hours. All presentations received a specialist CAMHS consultation. Over $50 \%$ required admission to a paediatric ward and $80 \%$ required onward referral to specialist CAMHS.

Clinical implications There is a need for a 24-hour specialist CAMHS and this allows increased rates of specialist assessment and onward referral for a high-risk group.

Declaration of interest All authors work in a specialist liaison psychiatry service.
The National Service Framework (NSF) outlined reasons that children and adolescents may require emergency or 'out-of-hours' mental health assessment. These included the rapid development of serious life-threatening conditions such as psychosis or suicidality, the development of urgent mental health difficulties while awaiting routine care or caregivers requiring urgent support. ${ }^{1}$ Ideally, specialist child and adolescent mental health services (CAMHS) would be available to provide emergency assessments during and outof-working hours; however, providing these services can be challenging for CAMHS in the context of limited resources and personnel. Despite many recommendations regarding the provision of 'out-of-hours' CAMHS, such services are still not available in parts of Ireland and the UK. ${ }^{1-5}$

The assessment of children's and adolescents' mental health is a challenging task, requiring considerable training and expertise. Emergency mental health assessments pose particular challenges, and clinicians must have knowledge about how a child's cognitive development has an impact on the assessment and management of risk. ${ }^{6,7}$ Assessments must include information from collateral sources, in particular the young person's caregiver. When formulating a management plan, the clinician must be aware of the caregiver's ability to support the child, child protection guidelines and procedures, and local pathways of care to facilitate appropriate onward referral. ${ }^{8}$ In 2000, significant concerns were raised about the ability of inadequately trained staff to provide these assessments. A study reviewing the quality of psychosocial assessments of selfharm in young people conducted by non-specialist doctors within emergency departments throughout the UK revealed that assessments were of variable quality, focused on shortterm risk and had low rates of onward referral (approximately $40 \%$ ) to specialist mental health services for further assessment. ${ }^{\text {? }}$

In light of these concerns, it has been recommended that where out-of-hours CAMHS assessments are not available, joint protocols must be agreed between specialist CAMHS, adult psychiatric services and paediatricians to ensure all children and adolescents receive the best possible care. ${ }^{5}$ Specific guidance on the management of self-harm in children and young people has been provided by the National Institute for Health and Clinical Excellence (NICE), including the need for practitioners to be experienced in the assessment of children and adolescents who self-harm, and to have received appropriate training in the assessment of mental capacity, consent and matters of child protection. It is also recommended that children and young people should normally be admitted overnight under the care of a paediatrician to facilitate assessment by specialist CAMHS the following day. ${ }^{8}$ Following the NSF report, the UK government made the provision of comprehensive CAMHS as one of the government's key priorities. Achieving widespread provision of specialist CAMHS $24 \mathrm{~h}$ a day, 7 days a week ('24/7') was one of the three key target outcome measures chosen to monitor 
progress towards this goal. ${ }^{10}$ However, the lack of 'out-of-hours' specialist CAMHS leads to a lack of evidence on the demand for or effectiveness of out-of-hours specialist CAMHS provision to guide service development. ${ }^{5}$ To date, significant gaps in service provision and information remain. We could find no published articles describing the demands or experiences of a 24/7 specialist CAMHS, or how models of service provision have been implemented in clinical practice within Ireland or the UK. In light of these challenges, we aim to provide information on such a service delivered over a 6-year period in Dublin, Ireland. This paper has two aims: to describe the model of service provision and to describe the demand for and outcome of emergency assessments over that time period.

\section{Method}

\section{Setting}

The Children's University Hospital is a tertiary referral teaching paediatric hospital in the centre of Dublin, Ireland. The emergency department within the hospital is one of the busiest in Europe and treats children and adolescents up to 16 years of age.

\section{Design and procedure}

This study used a retrospective case study design. As a result of a marked increase in the number of psychiatric presentations to the emergency department a centralised database was established in 2002, where standardised information on all emergency presentations with psychiatric symptoms was recorded. All presentations between 2002 and 2008 were reviewed and analysed. To ensure the accuracy of information, random cross checks were conducted whereby database entries were compared with original case notes. The information from the database was then coded and transferred to SPSS version 14 for Windows for statistical analysis.

\section{Participants}

There were 468 presentations to the emergency department with psychiatric symptoms over the 6-year study period.

\section{Ethical considerations}

Permission for the study was granted by the Children's University Hospital Ethics Committee for research. Parents/carers consented to the use of information provided by them in anonymous audit and research studies.

\section{Results}

\section{Model of service provision}

In line with best practice guidelines, a multi-agency protocol has been agreed between the catchment area specialist CAMHS, local adult mental health services and the paediatric service within the Children's University Hospital (CUH). The CUH provides emergency department services, paediatric in-patient and out-patient services and a multidisciplinary mental health liaison team (MHLT), which is a part of a larger department of child and adolescent mental health within the hospital. A consultant and senior registrar (specialty trainee year 4-6 equivalent) in child and adolescent psychiatry, two senior psychiatric social workers and two clinical nurse managers who have specialised in child and adolescent mental health make up the MHLT. Once a referral is received the team works together to provide a comprehensive multidisciplinary assessment. The senior registrar will conduct a psychiatric assessment and mental state examination of the young person, while the social worker and nurse will meet with the family to explore systemic factors, collect collateral history and liaise with outside agencies as appropriate. The consultant provides overall case supervision and assessment/input as required.

The adult mental health service provides emergency mental health assessments for 16- and 17-year-olds presenting to the emergency department of the local general university hospital (which provides services for those aged 16 upwards). During working hours, the CAMHS responds to requests for emergency assessments from existing service users and families, and/or from referral agencies if possible. The CUH emergency department facilitates emergency assessments of children up to 16 years with psychiatric symptoms on a 24 -hour basis. The CUH policy states that no child with psychiatric symptoms may be discharged from the emergency department without a specialist mental health assessment. This is facilitated by the CUH MHLT, supported by an 'on-call' rota staffed by consultants $(n=8)$ and psychiatric trainees $(n=8)$ who work in either the catchment specialist CAMHS or the department of child and adolescent mental health within the CUH. In line with NICE guidance, it is recommended that young people who present with self-harm or significant suicidal ideation should generally be admitted under a paediatrician to allow further medical and psychosocial assessment and discharge planning the following day.

The CUH MHLT ensures the guidelines are consistently adhered to. During working hours the team coordinates the provision of multidisciplinary mental health assessments. Each morning a MHLT liaison nurse reviews all emergency presentations over the previous $24 \mathrm{~h}$ with emergency department staff, providing a mechanism of ongoing audit to ensure all children who present with psychiatric symptoms receive a specialist mental health assessment, as well as providing support and psychoeducation to emergency department staff regarding psychosocial presentations. For children admitted with psychiatric symptoms, the team provides daily psychosocial assessments, ongoing liaison with paediatric and nursing staff and discharge planning. For young people who present out of hours and are not admitted, the MHLT reviews the psychiatric assessment notes and makes contact with the family the following day, as well as ensuring correspondence is sent to the family's general practitioner (GP) and an appointment date for follow-up by community CAMHS is obtained. The interagency policy ensures that children or adolescents who reside within the local CAMHS catchment area will be prioritised within the CAMHS for further review. 


\section{Demand for services}

There were 468 presentations to the emergency department with psychiatric symptoms over the 6-year period, with 35 presentations in 2002, increasing to 136 presentations in 2008 (Fig. 1). All children and adolescents and their families who presented received a specialist CAMHS consultation to inform onward management from the emergency department. Peak incidences were seen during late winter/spring months (January to April), and lowest incidences occurred during the school summer holiday period of July and August.

\section{Age and gender}

Presentations ranged in age from 5 to 18 years, with a mean age of 13 years; $20 \%$ of individuals $(n=96)$ were aged 12 years or younger at presentation. During the 6-year period, nine young people aged 16 and older presented to the emergency department and required review prior to discharge or transfer to the adult emergency department. Overall, females outnumbered males by a ratio of $6: 4$, however in the under-12 group males outnumbered females by a ratio of 2:1.

\section{Reasons for presentation}

The primary reason for presentation was recorded for each attendance $(n=468)$. Self-harm was the most common reason for presentation $(58 \%, n=271)$ followed by suicidal ideation $(27.8 \%, n=130)$ and drug and alcohol misuse $(n=34)$. Emotional (low mood or anxiety) and behavioural difficulties were far less common reasons for acute presentation $(n=16)$. Other reasons for presentation included acute psychotic symptoms $(n=7)$, anorexia nervosa and pseudoseizures.

\section{In working hours $v$. out of hours}

The time of presentation was recorded for $95 \%$ of individuals $(n=445)$. Only $41 \%$ of individuals presented between $09.00 \mathrm{~h}$ and $17.00 \mathrm{~h}$, with two-thirds $(n=301)$ presenting outside of standard working hours when weekends and bank holidays were considered. Unexpectedly, the highest rates of presentations occurred midweek and the lowest rates at weekends.

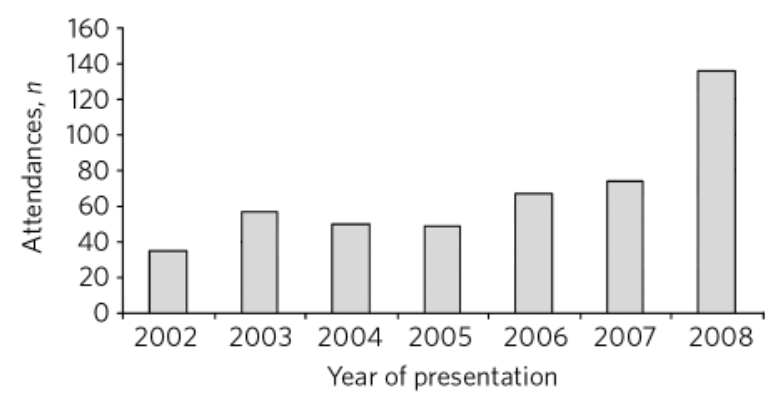

Fig 1 Number of attendances per year.

Significant differences existed between presentations during working hours and those out of hours. Children (12 years or less) were significantly more likely to present during working hours. In keeping with this finding, out-ofhours presentations showed a higher female:male ratio of 2:1 compared with working hours (female:male 1.2:1). Presentations of self-harm, suicidal ideation and drug or alcohol misuse were significantly more likely to occur out of hours, whereas other presentations such as low mood, anxiety and behavioural difficulties were more likely to occur during working hours. Of the seven young people who presented with psychotic symptoms, four presented outside of working hours. Overall, $52 \%(n=240)$ required admission to a general paediatric ward, and those who presented out of hours were significantly more likely to require admission. The most common reasons for admission were self-harm $(n=155)$ and suicidal ideation $(n=52)$. These results are summarised in Table 1.

Of those who required admission, the vast majority were discharged after one in-patient bed day, however there was a broad range (1-28 days) resulting in an overall mean stay of 3.3 days, shown in Fig. 2. Those who were admitted during working hours demonstrated a slightly longer mean stay (4.5 v. 3 days), but this difference did not reach statistical significance $(t(221)=-1.95, P=0.05)$.

Information on onward referral has been recorded for presentations since $2006(n=278)$. Of this sample, $80 \%$ of emergency presentations $(n=240)$ were referred for ongoing specialist CAMHS follow-up, with nearly twothirds of this subgroup being referred to the local specialist CAMHS.

\begin{tabular}{|c|c|c|c|c|}
\hline & During working hours & $\begin{array}{c}\text { Outside of working } \\
\text { hours }\end{array}$ & $\chi^{2}$ & $P$ \\
\hline Presentation, $n$ & 144 & 301 & - & - \\
\hline Presentations aged 12 or younger, $\%$ & 27.1 & 16.9 & 6.2 & $0.01 *$ \\
\hline Ratio, females:males & $1.2: 1$ & $2: 1$ & 4.05 & $0.04^{*}$ \\
\hline Admitted as in-patient, $\%$ & 36.4 & 59 & 19.8 & $<0.001^{* *}$ \\
\hline $\begin{array}{l}\text { Reason for presentation, \% } \\
\text { Self-harm, suicidal ideation or drug and alcohol misuse } \\
\text { Other presentations }\end{array}$ & $\begin{array}{l}89 \\
11\end{array}$ & $\begin{array}{c}96 \\
4\end{array}$ & 16.32 & $<0.001^{* *}$ \\
\hline
\end{tabular}

${ }^{\star} P<0.05,{ }^{\star \star} P<0.001$ 


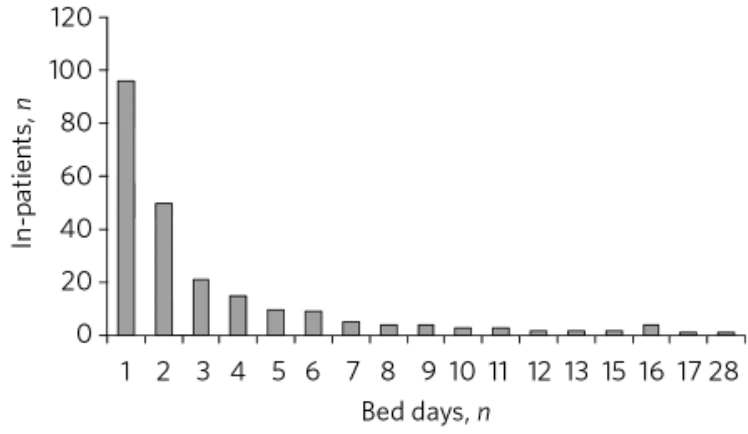

Fig 2 Number of in-patient bed days $(n=240)$.

\section{Discussion}

\section{Model of service}

Many different models of 24-hour service provision exist, for example the provision of an on-call system, where psychiatric review is provided as required during and out of hours using a rota system with staff from local CAMHS. Such a service may be based within local CAMHS offices or within a paediatric emergency department. Another model includes the routine admission of young people who present to the emergency department with psychiatric symptoms to facilitate further psychiatric assessment the following day from a psychiatric liaison service.

The model of 24-hour service we have described combines a psychiatric 'on call' with a paediatric liaison model of service delivery, combining the advantages of both service models. From the data collected it would be difficult to envision how we could have provided an effective 24-hour service through CAMHS alone without the support of paediatric colleagues, as self-harm and drug and alcohol misuse accounted for approximately two-thirds of presentations. Of those who presented with self-harm, an overdose with medication was the most common method used $(n=159)$, necessitating medical review. In addition to medical expertise the model provided an option for emergency admission, which would be otherwise limited in Ireland.

We believe that the multidisciplinary MHLT is central to the successful delivery of this model of service. During working hours, the MHLT provides a comprehensive multidisciplinary assessment with the minimum of delay, as assessments of the young person and family and the seeking of collateral history or liaison with nursing and medical staff can occur simultaneously. Working as a team prevents unnecessary duplication of assessment that may occur if social assessments and psychiatric assessments are undertaken by differing departments, and allows case discussion and management to be coordinated at team level under the supervision of a consultant child and adolescent psychiatrist. The presence of an onsite MHLT has allowed the team to build experience and expertise in the particular challenges associated with emergency presentations, and provides a mechanism to ensure follow-up case management for young people who present and are discharged out of hours. The daily attendance of the MHLT to the emergency department and the wards to review and discuss presentations over the previous $24 \mathrm{~h}$ provides support and psychoeducation to paediatric staff, and provides a mechanism to rapidly identify and address any challenges faced by paediatric staff in the management of young people with psychiatric difficulties.

A key factor in the success of inter-agency working is the ability of the stakeholders to communicate and develop positive working relationships. The efficient running of the MHLT has fostered positive and respectful relationships with paediatric colleagues, which in turn has created a positive ethos and approach within the hospital towards young people with psychiatric difficulties who present or require emergency admission. This has been particularly helpful in the light of limited in-patient capacity in Ireland, as in 2006 there was a total national provision of 10 public and 12 private child and adolescent psychiatric in-patient beds, serving a population of 4.2 million, with $25 \%$ under 18 years of age. ${ }^{11,12}$ Addressing this deficit in resources has been highlighted as a priority by the Irish government ${ }^{13,14}$ and capacity has now significantly increased, however, it remained low throughout the study period. The provision of a 24-hour mental health service to the $\mathrm{CUH}$ benefits community CAMHS in managing risk by having a safe, identifiable location to provide out-of-hours reviews with medical support, while facilitating this service within the CUH provides paediatric colleagues with 24-hour access to specialist mental health review and consultation. For the MHLT, the participation of CAMHS in 24-hour service provision ensures close working relationships with community CAMHS and minimises delays in discharge planning. Finally, ensuring the provision of a 24-hour specialist CAMHS assessment for young people up to 16 years of age assisted in formulating agreements with adult mental health services to provide emergency assessments for those aged 16 years or older during a time of transition of service delivery for this age group.

\section{Demand and outcome}

This study revealed a significant and rapidly increasing demand for emergency mental health assessments from our service, increasing fourfold during our study period. Of concern, $80 \%$ relate to self-harm and suicidal ideation. Self-harm is the strongest predictor for eventual suicide in both adolescent and adult populations. ${ }^{15-19}$ Community surveys estimate that $9 \%$ of Irish adolescents have engaged in self-harm ${ }^{20}$ and in 2006 it was estimated that 1 in every 165 Irish adolescent girls was treated in hospital as a result of self-harm. ${ }^{21}$ Two-thirds of our presentations occurred out of hours, and these individuals were significantly more likely to be part of this high-risk group. Our study also demonstrated a higher than expected rate (20\%) of childhood presentations (12 years or younger). Children and adolescents differ with respect to their physical, sexual, cognitive and social development, ${ }^{22}$ and children present significant challenges in assessment for emergency department or other non-specialist staff, particularly relating to presentations of self-harm or suicide. ${ }^{6,7,23,24}$

The reason for the rapid increase in demand during the study period requires review. In Ireland there are no studies monitoring the rates of child and adolescent psychiatric morbidly during the study period. In 2002, the National 
Suicide Research Foundation (NSRF) established the National Registry of Deliberate Self-harm, which monitors presentations of self-harm to emergency departments nationwide. This has shown national rates of self-harm (all ages) to be relatively stable between 2002 and 2006, with small increases in 2007 and 2008 ( $+4 \%$ and $+6 \%$ respectively). ${ }^{25}$ Significant regional variation was noted in 2007 and 2008, with the highest rate of presentation of self-harm recorded in the Health Service Executive Dublin/ North-East region, where our study was conducted. ${ }^{25}$ However, these rates of increase could not account for the fourfold increase in presentations. In 2006 the CUH changed its upper age limit guidelines for emergency presentations from 14 to 16 years. This does not fully account for the rise either, as 14- and 15-year-olds were seen by the service in all years of the study period, and accounted for nearly half of presentations between 2002 and 2005, rising to just over six out of ten presentations in 2006-8. A real increase in presentations of under $14 \mathrm{~s}$ was also seen, with 54 under 14 s presenting in 2008 alone, a figure greater than the total seen in three out of the four years from 2002 to 2005. The study was conducted during a period of economic prosperity in Ireland, and was not characterised by decreases in standards of living or the closure of other emergency assessment services or reductions in available psychiatric services for children and young people. One possible contributing factor to the increase in demand may be that the provision of a specialist and effective service may have encouraged more families or GPs to refer young people with psychiatric morbidity to the CUH emergency department for review, supported by ongoing national mental health awareness and suicide prevention campaigns targeted at young people in the community. ${ }^{26}$ This possibility urgently requires further evaluation, given the public health importance of increasing accessibility of CAMHS to those who self-harm.

\section{Effectiveness}

Currently there are no agreed outcome measures or models to allow us to establish the 'effectiveness' of our service, but our study provides encouraging results. One key marker of an effective service is its accessibility to those that require it. All children/adolescents and families who presented received a specialist CAMHS consultation to inform onward management from the emergency department $(n=468$, $100 \%$ ), compared with the $40 \%$ rate of referral of young people to specialist services by emergency department personnel previously reported. ${ }^{9}$ Without a 24 -hour model of service provision, up to two-thirds of presentations may not have accessed this specialist psychosocial assessment, despite the broad range of ages presenting (5-18 years) and the high risk associated with many of the presenting symptoms. Over half of emergency presentations still required admission to a paediatric ward for further management, and approximately four out of five presentations required referral to specialist CAMHS for ongoing follow-up. The rates of admission could not be explained purely on the basis of NICE guidance on self-harm, as four out of ten young people who presented with self-harm were able to be discharged from the emergency department following review by the service. It is also unlikely that this finding could be explained by a low threshold of onward referral, as the vast majority of onward referrals were to the local CAMHS who participate in the provision of the emergency service, and the on-call consultant child and adolescent psychiatrist routinely reviewed presentations during the weekend period. Overall, these figures indicate that emergency presentations are associated with high levels of psychiatric morbidity, warranting access to specialist review and ongoing attendance at specialist CAMHS.

Other markers of the efficacy of a service include the quality and cost of the service provided. Unfortunately, direct measures of quality and cost lie outside our study design. However, it is worth noting that without the provision of a 24-hour specialist CAMHS all young people who presented with self-harm would have required admission to facilitate further assessment, resulting in at least an additional 116 admissions during the study period. This has significant cost implications for paediatric hospitals and service commissioners, as well as placing increased demands on hospitals in terms of bed occupancy and availability of nursing staff in terms of risk management pending psychosocial review.

\section{Strengths and limitations}

Our study has limitations. Although assessments were conducted by staff working within specialist CAMHS, we have no markers to indicate the quality of assessments provided, or levels of satisfaction with the service model from service users. Also, our study design provides no method to compare levels of morbidity of our study population with those presenting through routine care in the community. Strengths of this study include that it was conducted in a 'real-world' setting. In common with many specialist CAMHS in Ireland and the UK, our service faces significant challenges relating to inadequate resources at community and tertiary CAMHS provision level. Our study has a large sample size, with information recorded over a significant period of time (6 years). Data were collected prospectively on all presentations during the period reviewed, preventing selection bias regarding cases or information recorded within our sample. Finally, our study is an important contribution to information in an area regarding service planning and development where available evidence and information is currently limited.

\section{Implications}

Providing a 24-hour CAMHS is a challenging task, requiring effective multi-agency collaboration and joint policy formation at a local level. Our study confirms that there is a significant demand for 24-hour services, with up to two-thirds of emergency presentations occurring out-of-working hours. Emergency presentations are associated with significant psychiatric morbidity, and the majority require admission for further specialist psychosocial assessment and/or specialist CAMHS follow-up. The most common reasons for presentation are self-harm and suicidal ideation, carrying the highest risk for future self-harm and suicide. 
Assessing child and adolescent mental health requires significant expertise and training, and non-specialist assessment has been demonstrated to be of poor quality, focused on short-term risk and associated with low referral rates to specialist services. In light of these factors and that the majority of presentations occur out-of-working hours, services must continue to focus on developing innovative solutions to providing 24-hour specialist CAMHS. We must continue to advocate and lobby for improved resources to provide the much needed capacity to provide this vital service for children and their families. Finally, further study and research should be conducted to evaluate differing models and effectiveness of 24-hour service provision to guide future service development.

\section{About the authors}

Patricia Byrne is a consultant child and adolescent psychiatrist at St Frances Clinic Psychiatric Liaison Service, Children's University Hospital, Dublin. Lorna Power is a research psychologist at University College Dublin. Carole Boylan is a clinical nurse specialist at St Frances Clinic, Children's University Hospital, Dublin. Mohammed Iqbal is a consultant child and adolescent psychiatrist at the Mater Child and Adolescent Mental Health Service in Dublin. Margo Anglim is the clinical director at St Frances Clinic, Children's University Hospital, Dublin. Carol Fitzpatrick is a professor in child and adolescent psychiatry at University College Dublin and a consultant child and adolescent psychiatrist at St Frances Clinic, Children's University Hospital, Dublin, Ireland

\section{References}

1 Department of Health, Department for Education and Skills. National Service Framework for Children, Young People and Maternity Services. Department of Health, Department for Education and Skills, 2004

2 Royal College of Psychiatrists. Acute In-patient Psychiatric Care for Young People with Severe Mental Illness. Recommendations for Commissioners, Child and Adolescent Psychiatrists and General Psychiatrists (Council Report CR106). Royal College of Psychiatrists, 2002.

3 Irish College of Psychiatrists. A Better Future Now. Position Statement on Psychiatric Services for Children and Adolescents in Ireland. The Irish College of Psychiatrists, 2006.

4 Department of Health and Children. A Vision for Change. Report of the Expert Group on Mental Health Policy. Stationery Office, Dublin, 2006.

5 Royal College of Psychiatrists. Building and Sustaining Specialist Child and Adolescent Mental Health Services (Council Report CR137). Royal College of Psychiatrists, 2006.

6 Jacobsen LK, Rabinowitz I, Popper MS, Solomon RJ, Sokol MS Pfeffer CR. Interviewing pre-pubertal children about suicidal ideation and behaviour. J Am Acad Child Adolesc Psychiatry 1994; 33: 439-52.

7 Pfeffer CR. Childhood suicidal behavior. A developmental perspective. Psychiatr Clin North Am 1997; 20: 551-62.
8 National Institute for Health and Clinical Excellence. Self-Harm: The Short-Term Physical and Psychological Management and Secondary Prevention of Self-harm in Primary and Secondary Care. Clinical Guideline 16. NICE, 2004

9 Hurry J, Storey P. Assessing young people who deliberately harm themselves. Br J Psychiatry 2000; 176: 126-31.

10 British Medical Association. Child and Adolescent Mental Health. A Guide for Health Care Professionals. BMA Board of Science, 2006.

11 Health Service Executive. Report of the Health Service Executive Service Forum on Child \& Adolescent Psychiatric In-Patient Capacity - Report 1 of 2. Stationary Office, Dublin, 2006.

12 Central Statistics Office. Census 2006. Stationery Office, Dublin, 2007

13 Health Service Executive. HSE National Service Plan 2007. HSE, Ireland, 2007 (http://www.hse.ie/eng/services/Publications/corporate/ HSE_National_Service_Plan_2007.pdf).

14 Health Service Executive. HSE National Service Plan 2008. HSE, Ireland, 2008 (http://www.hse.ie/eng/services/Publications/corporate/ National_Service_Plan_2008.pdf).

15 Goldacre M, Hawton K. Repetition of self-poisoning and subsequent death in adolescents who take overdoses. Br J Psychiatry 1985; 146 395-8.

16 Sellar C, Hawton K, Goldacre MJ. Self-poisoning in adolescents Hospital admissions and deaths in the Oxford region 1980-85. $\mathrm{Br} J$ Psychiatry 1990; 156: 866-70.

17 Hall DJ, O'Brien F, Stark C, Pelosi A, Smith H. Thirteen-year follow-up of deliberate self-harm, using linked data. $\mathrm{Br} J$ Psychiatry 1998; 172: 239-42.

18 Hawton K, Zahl D, Weatherall R. Suicide following deliberate self-harm: long-term follow-up of patients who presented to a general hospital. Br J Psychiatry 2003; 182: 537-42.

19 Zahl DL, Hawton K. Repetition of deliberate self-harm and subsequent suicide risk: long-term follow-up study of 11583 patients. Br J Psychiatry 2004; 185: 70-5.

20 Sullivan C, Arensman E, Keeley HS, Corcoran P, Perry IJ. Young People's Mental Health. A Report of the Findings from the Lifestyle and Coping Survey. The National Suicide Research Foundation, 2004.

21 National Office for Suicide Prevention. Annual Report 2007. HSE, Ireland, 2008

22 Meltzer H, Gatward R, Goodman R, Ford F. Mental Health of Children and Adolescents in Great Britain. TSO (The Stationery Office), 2000.

23 Tishler CL, Staats Reiss N, Rhodes AR. Suicidal behavior in children younger than twelve: a diagnostic challenge for emergency department personnel. Acad Emerg Med 2007; 14: 810-8.

24 Sarkar M, Byrne P, Power L, Fitzpatrick C, Anglim M, Boylan C, et al. Are suicidal phenomena in children different to suicidal phenomena in adolescents? A six year review. Child Adolesc Ment Health 2010; 15 197-203.

25 National Suicide Research Foundation. Annual Report 2008. The National Suicide Research Foundation, 2009.

26 National Suicide Review Group (NSRG). Reach Out - Irish National Strategy for Action on Suicide Prevention 2005-2014. National Suicide Review Group, 2005. 